Cahiers d'Études Germaniques

CAHIERS D'ÉTUDES

GERMANIQUES

$76 \mid 2019$

Émigration et mythe

\title{
Fred Wander : un Schlemihl viennois en exil en
}

\section{France}

Fred Wander: ein Wiener Schlemihl im französischen Exil

Fred Wander: a Viennese Schlemihl in French Exile

\section{Alfred Prédhumeau}

\section{(2) OpenEdition}

Journals

Édition électronique

URL : http://journals.openedition.org/ceg/4961

DOI : $10.4000 /$ ceg. 4961

ISSN : 2605-8359

Éditeur

Presses Universitaires de Provence

Édition imprimée

Date de publication : 2 mai 2019

Pagination : 223-236

ISBN : 979-10-320-0214-8

ISSN : 0751-4239

Référence électronique

Alfred Prédhumeau, "Fred Wander : un Schlemihl viennois en exil en France », Cahiers d'Études

Germaniques [En ligne], 76 | 2019, mis en ligne le 02 novembre 2020, consulté le 25 janvier 2021. URL : http://journals.openedition.org/ceg/4961 ; DOI : https://doi.org/10.4000/ceg.4961

Tous droits réservés 


\title{
Fred Wander : un Schlemihl viennois en exil en France
}

\author{
Alfred PRÉDHUMEAU \\ Université Toulouse-Jean Jaurès, CREG (EA 4151)
}

\begin{abstract}
Ich war zwanzig Jahre alt, ziemlich weltfremd, und wenn ich heute nach so langer Zeit daran zurückdenke, war es unter den verschiedenen Stationen meines Lebens eine der schönsten. Du kommst in Paris an, hast keine Ahnung von der wirklichen Welt, kein Gepäck, kein Geld, kennst die Sprache nicht, kennst keinen einzigen Menschen. Aber was macht das aus, wenn schon auf der Fahrt dorthin einer seine Knackwurst mit dir teilt ${ }^{1}$.
\end{abstract}

Dès la première page de ses mémoires, Wander donne le ton : une relation spécifique est en train de se mettre en place entre l'auteur et la France, pays qui l'ignore largement aujourd'hui encore, son œuvre ${ }^{2}$ n'ayant à ce jour été traduite en français, alors que son principal texte, Der siebente Brunnen ${ }^{3}$, est certainement un écrit littéraire majeur de langue allemande sur la Shoah. Ce texte fut publié au début des années 70 lorsque l'auteur put se remémorer l'horreur de son expérience personnelle. Itinéraire concentrationnaire difficile à reconstruire car il n'y pas de biographie complète de Fred Wander ${ }^{4}$, et il n'est malheureusement pas possible de combler

1. Fred Wander, Das gute Leben oder Von der Fröhlichkeit im Schrecken. Erinnerungen, Göttingen, Wallstein Verlag, 2006, p. 7. Cité ci-dessous : Leben. Nous utilisons 1'édition de 2006, enrichie par rapport à celle de 1997 .

2. Les ouvrages suivants de Fred et Maxie Wander, cités par ordre chronologique de la première édition, ont été consultés pour cet article. Fred Wander : Doppeltes Antlitz: Pariser Impressionen, Berlin, Verlag Volk und Welt, 1966; Nicole, Berlin, Verlag Neues Leben, 1971; Der siebente Brunnen, avec une postface de Ruth Klüger, Göttingen, Wallstein Verlag, 2005 [1971 : Berlin, Aufbau Verlag]; Ein Zimmer in Paris, Berlin, Aufbau Verlag, 1983 [1975]; Provenzalische Reise, Leipzig, VEB F.A. Brockhaus Verlag, 1978 [cosigné avec Maxie Wander]; Hôtel Baalbek, avec une postface de Erich Hackl, Göttingen, Wallstein Verlag, 2007 [1991: Berlin, Aufbau Verlag]; Das gute Leben oder von der Fröhlichkeit im Schrecken. Erinnerungen, Göttingen, Wallstein, 2006, éd. remaniée et complétée [1 $1^{\text {re }}$ éd. 1996, München, Hanser Verlag]. Maxie Wander: Tagebücher und Briefe, édité par Fred Wander, Berlin, Buchverlag der Morgen, 1979; Ein Leben ist nicht genug, édité et préfacé par Fred Wander, Frankfurt a. M., Suhrkamp, 2007.

3. Indications bibliographiques : voir note 2 .

4. Il y a bien une biographie en langue italienne : Alessandro Roveri, L'ebreo Fred Wander, straniero in patria, Milan, Angeli, 2009. L'apport de celle-ci est limité car l'auteur se contente de paraphraser en italien en rétablissant la chronologie de Leben de Fred Wander. Pour la période de la vie commune entre Maxie et Fred Wander, la biographie de Sabine Zurmühl, Das Leben, dieser Augenblick. Die Biographie der Maxie Wander, Berlin, Henschel Verlag, 2001 est certainement utile, malgré son style souvent trop journalistique et les violents conflits, y compris juridiques, qui opposèrent l'auteure à Fred Wander pour des passages, à son avis, diffamatoires car contraires à la vérité. 
cette lacune ici. Rappelons simplement qu'il vint en France en 1938, afin de fuir l'annexion de l'Autriche par l'Allemagne nazie, mais qu'il fut livré aux Allemands par le régime de Vichy en 1942. Déporté à Auschwitz, puis dans différents camps d'esclavage des SS, il a été libéré à Buchenwald en 1945 par l'armée américaine. Il souhaitait alors revenir en France, mais n'y parvint pas et s'installa à Vienne où il travailla comme reporter pour la presse communiste. De ce fait, il fut délégué au premier cours du Literaturinstitut ${ }^{5}$ à Leipzig qui venait d'être créé. Il s'installe définitivement en RDA après avoir épousé en 1956 Elfriede Brunner, plus connue sous le nom de Maxie Wander. Une grande partie de son œuvre est alors publiée en RDA et le Brunnen obtient le très réputé prix Heinrich Mann. Fred Wander parvient à vivre comme écrivain, faisant de la France une thématique majeure de son travail. Après que Maxie eut succombé à un cancer, il se réinstalle à Vienne en 1983, où il décède en 2006.

Juif, communiste, écrivain en RDA qui n'a pas fait partie des cercles d'opposition littéraire, au contraire de son amie Christa Wolf, il n'y eut de réception notable de son œuvre à l'Ouest, et, s'il y eut un bref regain d'intérêt après l'unification allemande, ses livres ont aujourd'hui disparu des étals des librairies et son $100^{\mathrm{e}}$ anniversaire en 2018 est passé, éditorialement parlant, inaperçu ${ }^{6}$. Pour la recherche, il en va différemment (nous donnons quelques exemples en note, sans vouloir être exhaustif) : depuis le changement de millénaire, il y a un intérêt accru en particulier pour ce qui dans son œuvre se rapporte à son expérience concentrationnaire ${ }^{7}$. Parfois Wander est inclus dans des études de littérature comparée, approche très productive de cette littérature mémorielle ${ }^{8}$. D'autres études vont se pencher sur la problématique de la fiction autobiographique ou de l'autobiographie fictionnalisée en se focalisant sur les problèmes de langue et de style que cela peut soulever ${ }^{9}$. Rares, cependant,

5. Institut für Literatur fondé en 1955 sur décision du Comité central du SED. L'Institut obtint un statut universitaire en 1958 et fut nommé Institut für Literatur Johannes R. Becher en 1959.

6. Cette année du $100^{\mathrm{e}}$ anniversaire fut l'occasion d'une conférence à Dortmund où la forte participation internationale a pu témoigner de l'intérêt croissant pour Fred Wander. Les actes en sont annoncés pour 2019. Walter Grünzweig, l'un des organisateurs de cette conférence, avait dirigé avec Ursula Seeber la publication d'un ouvrage collectif Fred Wander. Leben und Werk, Bonn, Weidle Verlag, 2005. Sans nul doute cette publication permet une excellente première orientation dans l'œuvre de Wander.

7. Signalons les travaux d'Andrea Reiter, en particulier : Auf daß sie entsteigen der Dunkelheit. Überlebensberichte ehemaliger KZ-Häftlinge, Wien, Löcker Verlag, 1995 et " "Was mich entmenschlicht hat ist Ware geworden, die ich feilhalte" : Concentration Camp Experience of Jean Améry and Fred Wander », in The Journal of Holocaust Education, vol. 5, nº 1, été 1996, p. 1-15 ainsi que Martine Benoît « "Nous ne mourrons pas, nous vivrons. Eux mourront”. Le Septième Puits de Fred Wander », in Revue d'Histoire de la Shoah 201, 2014/2, p. 545-558.

8. Voir Martine Benoît, « Comment (ne pas) dire le bourreau : deux romans de Jurek Becker et Fred Wander publiés en RDA », in Lieux et figures de la barbarie, Bruxelles, Lang, 2012, p. 329-338. Julien Aurélie, Errance identitaire, errance scripturale. Patrick Modiano, W. G. Sebald, Fred Wander et la littérature de l'après, thèse, Rennes 2, 2016. Ulrike Schneider, Jean Améry und Fred Wander: Erinnerung und Poetologie in der deutsch-deutschen Nachkriegszeit, Berlin, de Gruyter, 2013.

9. Agnieszka Rosik, «... wir wollenjazum Leben sagen ...»: Fred Wanders autobiographische Prosa, thèse, Wien, 2001 ; Serge Yowa, Eine Poetik des Widerstands: Exil, Sprache und Identitätsproblematik bei Fred Wander und Ruth Klüger. Beitrag zur neueren kulturwissenschaftlichen und fachübergreifenden 
sont les travaux qui ont pour objet l'expérience française de l'auteur ${ }^{10}$. C'est notre objectif de tenter d'apporter quelques éléments à cet aspect primordial du corpus littéraire de Wander ${ }^{11}$.

\title{
Wander, le Schlemihl autoproclamé
}

\begin{abstract}
« Weißt du denn nicht, wo du hingehörst, Itzig? » [...] Ich wußte seit jeher, du gehörst nicht dazu, du gehörst nirgends dazu! [...] Ich glaube, daß meine Herkunft und meine Kindheitserlebnisse so prägend waren - die Namen Rosenkranz oder Rosenstingl $\left[\ldots{ }^{12}\right]$ den Juden von korrupten deutschen Beamten in der Vorzeit aufgezwungen - daß diese Namen so befleckend und stigmatisierend waren, daß ich notwendig und eigentlich ohne Mühe und ohne Schmerz [...] eine Art Außenseiter-Identität zu entwickeln hatte ${ }^{13}$.
\end{abstract}

\section{Cette identité amène l'auteur à trouver son chemin, de paria et de Schlemihl :}

Das alles war mir damals noch nicht bewußt, aber mein Weg zeichnete sich bereits deutlich ab, der Weg des Paria, des Ausgestoßenen, der sein Schicksal annimmt, aber auch Widerstand leistet, auf seine passive vertrackte $\mathrm{Art}^{14}$ !

Et, écoutant un oncle raconter le sort des Juifs exclus de la société, il fait le constat d'une vérité déguisée en interrogation «Wer in unserem Umkreis war kein Schlemihl ? », vérité donc qui le définit autant que ses proches ${ }^{15}$.

Le Schlemihl est un personnage mythique de la tradition orale juive qui porte en lui la malchance tout en y trouvant son bonheur. Il est maladroit, c'est un benêt. On le reconnaîtra aisément à son comportement inadéquat, sa parole inadaptée, son accoutrement déplacé - c'est donc un paria ${ }^{16}$, un marginal, l'étranger par excellence. Il n'est donc pas étonnant que chez Wander ce modèle autobiographique, qui relève

Shoah-Autobiografieforschung, Würzburg, Königshausen \& Neumann, 2014; Jörg Thunecke, «Fred Wander's Semi-Autobiographical Narrative, The Seventh Well - "Such stories I never heard again" » in Nick Meyerhofer (éd.), The Fiction of the I: Contemporary Austrian Writers and Autobiography, Riverside, 1999, p. 242-58.

10. Thomas Keller, «Das teuflische und das gute Frankreich. Fred Wander », in Daniel Azuelos (éd.), Lion Feuchtwanger et les exilés de langue allemande en France de 1933 à 1941, Jahrbuch für Internationale Germanistik, Reihe A. Band 76, Bern, Peter Lang, 2006.

11. Nous renvoyons aussi à notre contribution aux actes de Dortmund provisoirement intitulée "Fred Wanders "französische Texte" », à paraître en 2019. En outre, nous préparons un travail monographique qui tentera d'éclairer de manière plus approfondie cet aspect de l'œuvre de Fred Wander.

12. Nous avons supprimé une liste d'une demi-douzaine de noms «typiquement juifs » que cite Wander - sans toutefois y inclure son patronyme de naissance « Rosenblatt». Il changea de nom en 1950 à Vienne.

13. Wander, Leben, p. 33/34.

14. Ibid., p. 47.

15. Ibid., p. 37.

16. Il n'est pas possible ici de tenter un résumé des théories sociologiques qui sont à l'origine de ces termes. Nous renvoyons à Max Weber, Le Judaïsme antique, Paris, Plon, 1970 ; Bernard Lazare, Le Fumier de Job, Strasbourg, Circé, 1990. Pour ce qui concerne le paria selon Arendt, voir Enzo Traverso, La fin de la modernité juive. Histoire d'un tournant conservateur, Paris, La Découverte, 2016, p. 79-105. 
d'un choix personnel souvent conscient, va fusionner avec celui d'Ahasvérus, celui du Juif errant ${ }^{17}$, présent à la fois dans la mythologie orale juive et dans la tradition chrétienne où il est instrumentalisé par la propagande antisémite. Ahasvérus est condamné à une pérégrination éternelle jusqu'au retour du Messie. Il était boutiquier sédentaire avant de devenir victime errante. Le Juif errant occupe dans la mythologie chrétienne une place ambiguë : d'un côté il n'a pas reconnu le rôle messianique de Jésus mais d'autre part, il est témoin oculaire de la passion du Christ ${ }^{18}$. Dans la tradition juive, il est la métaphore de la diaspora et celui qui reste fidèle à sa foi ${ }^{19}$. Comme l'a signalé Enzo Traverso, «Ahasvérus est devenu la métaphore d'une minorité vivant aux marges de la société, tantôt par choix, tantôt par contrainte ${ }^{20}$. »

Dans un texte longtemps oublié, pourtant essentiel pour la compréhension de l'identité juive d'émigration, Hannah Arendt dans We Refugees ${ }^{21}$, publié en 1943 à New York après sa fuite de France, a théorisé l'impact des concepts de Schnorrer, Schlemihl, Paria et Parvenü ${ }^{22}$ sur l'identité juive. Elle n'a cependant pas défini ces termes dans ce contexte - comme Wander, elle se contente de les utiliser, leur signification semblant tomber sous le sens, en cherchant à les confronter avant tout à la tentative désastreuse, selon elle, d'assimilation entreprise par les juifs d'Europe :

Unsere heute so verdächtige Loyalität hat eine lange Geschichte. Es ist die 150-jährige Geschichte des assimilierten Judentums, das ein Kunststück ohnegleichen vorgeführt hat: obwohl die Juden die ganze Zeit ihre Nichtjüdischkeit unter Beweis stellten, kam dabei nur heraus, dass sie trotzdem Juden blieben ${ }^{23}$.

Pour Hannah Arendt la référence idéologique pour la définition du paria est celle de Bernard Lazare qui, dans le Fumier de Jacob de 1927, avait dessiné le portrait du paria rebelle - Lazare était anarchiste.

Aber es stimmt gleichfalls, dass die ganz wenigen unter uns, die versucht haben, ohne all diese faulen Tricks der Anpassung und Assimilation ihren Weg zu machen, einen zu hohen Preis bezahlt haben: sie setzen die wenigen Chancen aufs Spiel, die sogar ein Vogelfreier in dieser

17. La notice relative à l'Histoire du Juif errant de Jean d'Ormesson propose un résumé utile de l'histoire de ce mythe. Cf. Jean d'Ormesson, Histoire du Juif errant, in Euvres, Paris, Gallimard, Bibliothèque de la Pléiade, 2015, p. 1608 sqq. Notons en passant que chez d'Ormesson, l'utilisation antisémite du personnage est occultée et que la Shoah est inexistante. Ce que certains critiques n'omettront pas de relever.

18. Cf. Marcello Massenzio, « Le Juif errant entre mythe et histoire. Trois variations sur le thème de la Passion selon le Juif errant », Annuaire de l'École pratique des hautes études (EPHE), Section des sciences religieuses, [http://asr.revues.org/106].

19. Chez d'Ormesson aussi, le Juif errant, Simon Fussgänger - qu'il eût pu appeler « Wanderer » - ne se convertit pas au catholicisme.

20. Traverso, La fin, p. 29.

21. Hannah Arendt, « We Refugees », Menorah Journal 31, n 1, janvier 1943, p. 69-77. Voir : [http:// amroali.com/2017/04/refugees-essay-hannah-arendt/]. J'ai utilisé la traduction allemande publiée la première fois en 1986 et rééditée récemment chez Reclam : Wir Flüchtlinge, traduction de Eike Geisel, avec un essai de Thomas Meyer, Stuttgart, Reclam, 2016. Voir aussi : Giorgio Agamben, «We Refugees », Symposium: A Quaterly Journal in Modern Literatures, vol. 49, n² 2, 1995, p. 114-115. Il existe une traduction française du texte d'Arendt accessible en ligne : [https://www.cairn.info/revuepouvoirs-2013-1-page-5.htm].

22. Arendt, Flüchtlinge, p. 21 et 33-35.

23. Ibid., p. 31. 
verkehrten Welt noch besitzt. / Die Einstellung dieser wenigen, die man Bernard Lazare zufolge als „,bewusste Parias“ bezeichnen könnte, lässt sich durch die jüngsten Ereignisse ebenso wenig erklären, wie die Haltung unseres Herrn Cohn, der mit allen Mitteln versucht, den Aufstieg zu schaffen $^{24}$.

Le Schlemihl de Wander, s'il est lui aussi « paria », n'est pas rebelle - ce pas-là, il ne le franchit point. Quant au statut de «parvenu », s'il faillit l'obtenir en RDA, grâce à sa biographie de victime du nazisme, son manque d'engagement positif en faveur du régime et son esprit réfractaire aux formatages idéologiques l'en empêchèrent ${ }^{25}-\mathrm{si}$ tant était qu'il l'eût recherché.

Wander avait adopté ce nom pour bien labelliser son errance identitaire. Partant de là, il va effectuer une jonction entre ce personnage fusionnel judéo-chrétien et le narrateur - qui lui se place dans la tradition hassidique, comme par exemple dans Baalbek ${ }^{26}$, et saura, comme chez Benjamin, donner un sens messianique à sa narration et donc, par extrapolation, à l'Histoire. Ce n'est pas un hasard si cet angle messianique va prendre l'aspect d'une utopie ${ }^{27}$, idée d'utopie qu'il précisera au fur et à mesure de ses lectures, parfois très éclectiques, en particulier de Camus - il ne fait aucun doute que la lecture de Camus ${ }^{28}$, de l'Étranger et de la Peste, trouvait une résonance correspondant à son expérience - et de Thoreau, le faisant évoluer d'un marxisme superficiel et assimilé sans enthousiasme vers un anticapitalisme « hippie » et ce qu'il pensait être la philosophie du taoïsme zen ${ }^{29}$.

Le Schlemihl de Wander est à la recherche de la Terre promise mythique, projet autant humain que politique, auquel il espère aboutir en transcendant les horreurs de l'«État français » et du Reich nazi. Ce Schlemihl-là est aux antipodes du protagoniste de la Schicksalsreise ${ }^{30}$ de Döblin, devenu Juif errant lui aussi, de manière tout à fait involontaire, qui va pendant la même période s'agenouiller à Mende devant le crucifix de la cathédrale, tourner définitivement le dos à son origine judaïque, ce que

24. Ibid., p. 34. Traverso donne un résumé de ces approches dans La fin de la modernitéjuive, en particulier pour ce qui concerne Lazare et Arendt : « Pour Bernard Lazare, le paria n'est pas seulement l'exclu ; il est le proscrit qui se transforme en rebelle, qui n'accepte pas de subir passivement sa condition d'opprimé et en fait le point de départ d'une révolte politique. [...] Aux antipodes du paria, et en quelque sorte sa doublure dialectique, se dessine la figure du parvenu, le juif qui essaie d'échapper à sa condition en la contournant, en l'exorcisant, en faisant semblant de l'ignorer, sans combattre ses oppresseurs mais en s'identifiant à eux par de multiples formes de mimétisme. » Voir Traverso, La fin, p. 84-85.

25. Le symbole de ce statut de parvenu potentiel est le « passeport rouge » dont il était porteur et qui lui permettait de passer la frontière entre la RDA et Berlin-Ouest - l'une des mieux gardées au monde, faut-il le rappeler ? - ou d'effectuer des voyages fréquents à l'Ouest, en Allemagne, en Autriche et en France.

26. Voir Wander, Baalbek, p. 183-184, où le personnage de Mendele correspond au modèle adopté.

27. Il n'est pas possible ici de débattre du terme d'« utopie ». Je renvoie à l'article de Gérard Raulet, «L'utopie est-elle un concept? », Lignes 1992/3, n 17, p. 102-117, [www.cairn.info/revue-lignes01992-3-page-102.htm].

28. Camus se trouve dans les listes des auteurs lus par Wander aux archives à Berlin.

29. Cette assimilation du taoïsme - ou ce qu'il pense être le taoïsme - par Wander ne peut être traitée ici.

30. Alfred Döblin, Schicksalsreise. Bericht und Bekenntnis, édition critique par Anthony W. Riley, Solothurn, Walter-Verlag, 1993; Voyage et Destin. Récit et confession, trad. par Pierre Gallissaires, Monaco, Éditions du Rocher, 2001. 
Sebald ne manquera de lui reprocher violemment ${ }^{31}$, et se convertir, sous le soleil californien, au catholicisme, alors que notre Schlemihl viennois se trouvera dans les wagons à bestiaux l'emmenant de Rivesaltes à Drancy et de Drancy à Auschwitz.

Le protagoniste wandérien n'est jamais un homme d'action. S'il a un projet politique et un objectif humain identifiables, il se contente d'observer, de photographier (mentalement d'abord), d'écouter, de raconter. Le contenu de cette imitatio est un présent décalé car il est impossible à notre narrateur de se replonger immédiatement à sa libération de Buchenwald dans l'horreur du vécu et ce n'est que peu à peu, par bribes, qu'il va se reconfronter à ses années d'exil et de camps de concentration. Il mettra vingt ans à se réapproprier son histoire et celle de ses compagnons de souffrance, sa narration postfactuelle a pour conséquence que son travail de mémoire est lui-même soumis à des fluctuations et des indéterminations chronotopiques qui rendent difficile, voire impossible, une réécriture de certains chapitres de sa biographie, dont il était le seul dépositaire. Cela est particulièrement vrai pour les étapes françaises de son périple de 1938 à 1942 - pour exemple : il situe l'offensive allemande décisive en France en $1941^{32}$ - ce que le lectorat en RDA eût pu corriger.

\section{Le Schlemihl en France}

Wander arrive en France en mai 1938 ; il prend le train de Lyon à Paris après un stage linguistique intensif à la prison de Pontarlier, où il apprend les fondements du français populaire et argotique sous les coups et les brimades de ses codétenus. Dans le train, il est subjugué par le comportement des autres voyageurs : « Die Leute blicken dir hier ins Gesicht, dachte ich und hatte auf einmal [...] das Gefühl von einem völlig neuen Leben ${ }^{33}$. » À Paris, gare de Lyon, il est immédiatement sous le charme de la ville. Et de ses habitants : « Die Szenerie betrachtend fühlte ich mich zum erstenmal mit allen Menschen eins [...]. Ich war ein Ausgestoßener, ein Paria aber ich gehörte dazu ${ }^{34}$. »

Le Schlemihl prend la mesure de la ville et des Français. Dès les premiers pas faits à la gare de Lyon, il sait qu'il est un Schlemihl, un paria. Même s'il ne va théoriser le concept que bien plus tard, lors de discussions à Paris avec une amie dont il rend compte dans Das gute Leben :

Natürlich [gibt] es auch unter Nichtjuden Tolpatsche und Schlemihle, aber der jüdische Nebbochant, wie die Juden sagen, ist der Archetyp des Schlemihls, denn er ist auch ausgestoßen, wenn er kein Schlemihl ist! Er steht außerhalb der Gesellschaft, seine Heimat ist nirgendwo. Er leidet an dieser Welt aber er genießt sie. Er ist ein verwundeter und gequälter Mensch, der aber

31. Voir W. G. Sebald, Der Mythus der Zerstörung im Werk Döblins, Stuttgart, Klett, 1980. Sebald voit dans la tentative de mener une existence d'assimilé à Berlin et de l'échec de celle-ci, la cause profonde de son apostasie. Voir p. 71 et suivantes. Sebald est parfaitement en phase avec le bref texte d'Arendt - qu'il ne connaissait probablement pas.

32. Wander, Zimmer, p. 87.

33. Wander, Leben, p. 7.

34. Ibid., p. 11. 
gelernt hat sein Unglück mit Abstand zu sehen und sogar mit Ironie. Er ist passiv, leistet keinen Widerstand, sondern zieht sich auf die letzte innere Barriere seines Menschseins zurück. Und das wiederum gibt ihm $\mathrm{Kraft}^{35}$.

On ne saurait exprimer plus clairement la distance qui sépare le Schlemihl wandérien du paria rebelle tel que le préconise Arendt ! Pour lui, le statut de Schlemihl est collectif, commun à tous les juifs. En effet, il fait dire à Sascha dans Baalbek, à la suite d'un long monologue relatif à l'existence de Dieu et à l'idolâtrie ${ }^{36}$, d'où le renvoi à Baal dans le nom de l'hôtel, que les juifs ne sont pas le peuple élu :

Die Juden sind keineswegs das ausgewählte Volk, wie verschiedene Eiferer behaupten, und wenn, dann vielleicht nur in dem Sinn, daß sie ein lebendiges Beispiel abgeben für das Anderssein in der Welt, das Fremde, das wir aber achten sollen, wie es in der Bibel steht ${ }^{37}$.

Le narrateur, à Marseille, dans cet hôtel ${ }^{38}$, se définit comme " ahnungslos, beschränkt ${ }^{39} »$, comme un « Nebbochant, nebbich, ein armer Mann, ein anständiger und schüchterner junger Mann ${ }^{40} »$ qui va faire la rencontre de Katja en faisant la queue pour une demande de visa ${ }^{41}$. Elle a un comportement détendu, ouvert :

Katja $^{42}$ benahm sich völlig ungezwungen im Umgang mit Menschen, diese vertrackte Ungezwungenheit und Vertrautheit der Entwurzelten, der Vertriebenen, die nichts zu verbergen, nichts mehr zu verlieren hatten, auf dem Grund angekommen, im Niemandsland der Parias ${ }^{43}$.

La jeune résistante prend en charge le Schlemihl, qui est bien incapable de résoudre les problèmes les plus simples auxquels il est confronté. Katja va l'aider à se tirer de situations dangereuses, comme d'autres protagonistes le font, parfois de manière intéressée, mais presque toujours avec efficacité. Néanmoins, la spirale de la malchance qui caractérise le Schlemihl suit son cours - et elle porte un nom : «Der Name Auschwitz hätte uns damals nichts gesagt [...]. Und doch war Auschwitz bereits Realität und so greifbar über unseren Köpfen wie jene grauen und lila Wolken am Himmel $[\ldots]^{44}$. »

Ce qu'il importe de noter dans ce texte, c'est qu'il concentre son récit autour de la tension - on est tenté de dire « dialectique »- entre notre Schlemihl, paria passif,

35. Ibid., p. 228.

36. Cette diatribe pourrait être lue comme réponse au choc «mystique » que Döblin décrit dans sa Schicksalsreise. Mais comme nous n'avons la preuve que Wander connaissait ce texte, nous ne pouvons que constater la divergence profonde entre ces auteurs.

37. Wander, Baalbek, p. 51.

38. L'hôtel est dans la littérature de l'exil allemande un topos métaphorique sur lequel nous reviendrons ailleurs.

39. Wander, Baalbek, p. 85.

40. Ibid., p. 87.

41. Évidemment, la scène de la file d'attente devant le consulat américain, ou tout autre consulat susceptible de délivrer un visa, fait partie des topoi de la littérature de l'exil germanophone.

42. Le personnage de Katja est taillé à l'image de Maxie. Ce n'est pas le lieu ici d'ouvrir le dossier essentiel de l'image des femmes chez Wander - prostituées, mères, compagnes, amies. Notons simplement que les descriptions en sont généralement saisissantes et marquées par une grande gratitude à l'égard des femmes qui l'ont aidé à vivre ou à survivre.

43. Wander, Baalbek, p. 95.

44. Ibid., p. 95-96. 
et Katja, paria résistante. Le personnage de cette jeune femme est à l'opposé du narrateur, alter ego de Fred : elle lutte, se bat, ne s'en laisse conter par les structures et les comportements machistes. Elle est un premier pas vers l'utopie concrète que cherche Wander sans qu'il ait été en mesure, en 1942, d'en définir les contours.

\title{
Vers une utopie paria-disiaque ?
}

Dans Der siebente Brunnen, il y a maints héros qui incarnent cette même volonté : vaincre la dictature nazie - et en filigrane toute dictature - et construire, après sa défaite, des sociétés meilleures dont Wander va tenter une définition dans Leben:

Wir leben in einer Welt, die sich selbst zerstört, wie viele von uns befürchten. Und einige glauben an eine Zeit und ein Leben danach und daß eine neue Welt im Entstehen ist, die aus dem Chaos hervortreten wird: Die eine Welt, eine Welt ohne Grenzen, ohne Ideologie, ohne Macht und Besitz, ohne diese korrupten Systeme der Oligarchie, die viele Länder dieser Erde beherrschen ${ }^{45}$.

L'auteur de ces lignes n'a pas de vision concrète de l'organisation d'une société future ; il sait ce qu'il ne veut pas, mais ne propose aucune alternative correspondant à une « utopie concrète » et, de même, n'a pas de moyen d'action à proposer pour sortir les sociétés contemporaines, socialistes ou capitalistes, de leurs impasses respectives.

Du reste, aussi bien dans Baalbek que dans Der siebente Brunnen, la grande majorité des protagonistes, aussi parmi ceux qui luttent dans les camps, vont périr. Les censeurs est-allemands du Brunnen ne s’y méprendront : «Die Arbeit ist nicht dem organisierten antifaschistischen Widerstand gewidmet, obwohl er an einigen Stellen spürbar wird ${ }^{46}$. » Et le second censeur remarque :

\begin{abstract}
Unerträglich wäre die Summe der Widerstandslosigkeit, die hier schonungslos an den Tag gebracht wird, stände nicht der Ich-Erzähler hinter allem mit ständigem Forschen eben nach dem Widerstand. Es gibt unter diesen Häftlingen kein Anzeichen für eine Selbstorganisation zur Abwehr gegen die Peiniger. Nicht einmal in Buchenwald werden diese Spätkommenden in ihren Quarantäneblocks von der Organisation der antifaschistischen Kämpfer erfasst. [...], all das wäre in der Summe von Fatalismus unerträglich, hätte nicht der Autor in einigen wenigen Gestalten - einem Pariser Maquisard, einem sowjetischen Kriegsgefangenen, einem noch unterm Galgen mannhaften Antifaschisten - Gegenpole geschaffen zu der geschilderten jüdischen Ausweglosigkeit ${ }^{47}$.
\end{abstract}

Ce censeur oublie de mentionner que l'action positive des antifascistes dans les camps est tout aussi désespérée que le fatalisme de certaines victimes juives (les deux tiers des susnommés périront), mais il s'agit de faire entrer le texte dans le moule de la doxa de l'héroïque antifascisme prolétarien. Wander ne manque cependant pas de souligner la courageuse intransigeance des suppliciés français : ce sont eux qui portent en eux le germe des victoires futures (et non le soldat de l'Armée rouge !).

45. Wander, Leben, p. 213.

46. Archives fédérales allemandes, DR1/2999a, p. 165.

47. Archives fédérales allemandes, DR1/2099a, p. 170. Cette expertise est de la plume la plus redoutée, car dogmatique-intransigeante, des censeurs du Ministère de la culture, Mme Gärtner Scholle - nous n’insisterons pas sur le reproche d'origine antisémite de la « jüdische Ausweglosigkeit ». 
Pour exemple Pépé du Brunnen, militant communiste qui fait l'apologie de la révolution antinazie :

[...] gute Menschen können wir jetzt nicht brauchen, wir brauchen Recken, Kämpfer, Kopfabschlager, Messerschleifer, Ausbrecher. / Ihr werdet alle Sorten von Leuten brauchen, sagte ich [le narrateur, N.D.A.], wenn die Revolution gemacht ist ${ }^{48}$.

Par cette citation est mis en exergue le potentiel révolutionnaire des résistants français. Ce potentiel est le premier palier de l'utopie wandérienne, sa réalisation ou sa mise en pratique est illustrée de manière métaphorique par le tailleur de pierre travaillant dans une des nombreuses carrières près de Montpellier où fut transféré notre Schlemihl-narrateur après son arrivée au camp d'internement d'Agde. Cette séquence est l'une des plus impressionnantes de Baalbek. Le narrateur et son ami Joschko échouent dans leurs tentatives à faire éclater une roche.

\begin{abstract}
Und nun war der große Augenblick gekommen da Martini den Hammer nahm. Er ging ruhig um den Steinbrocken herum, der ihm bis an die Brust reichte, und betrachtete ihn. Seine Augen hatten den gleichen Ausdruck wie vorhin, als er in unsere Gesichter blickte. Er schaute den Stein genau an, dann hob er den Hammer und schlug zu. Der Block flog auseinander, wie von einer Dynamitpatrone gesprengt. [...] // Martini hatte nun schon mehrere Blöcke zerlegt, sein Schlag mit dem Hammer wirkte jedesmal wie eine Ladung Pulver. Und dann fand er endlich ein paar Worte, was schwer sein muß für einen Mann, der selbst ein Stück von dem Berg geworden ist, den er zerbricht. Du mußt, sagte er, um den Stein herumgehen, ihn dir gut ansehen und den Punkt finden! - Das genügte. Wir waren doch nicht blöd. Wir hatten verstanden, daß der Stein einen schwachen Punkt hatte, den man entdecken mußte ${ }^{49}$.
\end{abstract}

C'est par cet acte « anarchiste », solitaire, violent que Wander pense qu'il devrait être possible d'anéantir la dictature fasciste - comme toute dictature ${ }^{50}$ ? Les autres actes relevés dans ces textes sont tout aussi isolés et coupés des masses (contraires donc à la théorie communiste de l'action révolutionnaire), même si émanant d'un petit groupe de conspirateurs. - Il s'agit de transport d'armes dans un corbillard, d'actes individuels de sabotage ou de feux de forêt menaçant des installations allemandes ou de la milice. Nous retiendrons de ce premier palier « utopique » que le narrateur n'est jamais impliqué directement, au mieux (ou au pire !), on l'utilise pour des fonctions subalternes sans même l'informer du contexte car, le moins il en sait... Nous noterons en outre que le taux de survie parmi ces résistants de la première heure (hors camps) est bien plus élevé que parmi les passifs résignés à se laisser faire et n'opposant aucune résistance, généralement pas même verbale. Rares sont en effet ceux qui, face à l'imminente déportation de Rivesaltes vers Drancy, osent élever la voix :

Endlich, die Nacht war gekommen, rief er [un officier français, N.D.A.] alle Versammelten näher heran: Morgen leite ich einen Transport, begann er herablassend freundlich, ich gebe Ihnen einen gutgemeinten Rat - machen Sie uns keine Schwierigkeiten [...] - Aber erlauben Sie, meldete

48. Wander, Brunnen, p. 84.

49. Wander, Baalbek, p. 189/190. La séquence « carrière » débute page 185 et se termine page 191.

50. Il ne fait aucun doute, à mon avis, que ce passage est une métaphore de l'action directe anarchiste - le prolétaire en question étant travailleur immigré italien, probablement réfugié antifasciste, originaire, peut-être, d'une carrière de Carrare, le haut-lieu de l'anarchisme italien. 
sich ein Gefangener sarkastisch zu Wort, wir machen Ihnen keine Schwierigkeiten. Sie liefern uns ans Messer und beklagen sich darüber, daß wir Ihnen Schwierigkeiten bereiten ${ }^{51}$ ?

Ailleurs, pour ainsi dire en contre-plan dans Baalbek, l'un des protagonistes anonymes lance la comparaison avec Job à une ronde occupée dans la chambre d'hôtel à trouver une réponse biblique au malheur qui les frappe :

Hiob, immer wieder Hiob ! / Warum machen sie das mit uns, warum läßt Gott es zu, daß sie das mit uns machen können, womit haben wir so schwer gesündigt ? [...] / Warum müssen die Unschuldigen mit Schuldigen büßen? / Gott hat Hiob vernichtet, seine Häuser zerstört, seine Viehherden wurden geraubt, die Söhne und Töchter erschlagen. Und Hiob hat den Tag verflucht, da er geboren wurde, und hat geschrien, warum? Hat aber keine Antwort bekommen. [...] / Aber das Leben kam zurück zu Hiob [...], es kam zurück auf seine Felder und in seine Wohnungen, er bekam alles zurück, aber keine Antwort. Nur das Leben selbst ist die Antwort ${ }^{52}$.

La réponse aux catastrophes causées par les dictatures est la vie même - cette réponse n'en est évidemment pas une, car il s'agira pour le narrateur de définir ce qu'est la bonne, la vraie vie, une vie hors oppression et aliénation ; ce projet, il pense l'avoir découvert en germe en France, en Provence. Cette vie, il imagine la réaliser grâce à une opposition «zen ${ }^{53}$ aux dictatures auxquelles il fut exposé : le nazisme et le socialisme stalinien; mais aussi dans une opposition intégrale au consumérisme de la modernité et aux aliénations que celle-ci génère.

Il faut, dans ces textes de Wander, faire la différence entre ce qui fut publié en RDA et ce qui parut ultérieurement en Allemagne après sa rémigration à Vienne après 1983 : certaines concessions idéologiques antérieures de la période 1958-1983 peuvent s'expliquer par le désir d'être publié et donc de vouloir passer le seuil de la censure en RDA. Encore qu'on ne sache exactement ce qui fut adhésion et ce qui fut pragmatisme opportuniste. Ce que l'on peut constater avec certitude, c'est que Wander ne fit nulle part l'apologie de la dictature du prolétariat mais que son projet de société repose sur une vision « hippie» du bonheur sur terre. Aux régimes oppresseurs de Vichy et de la société capitaliste, il oppose une vision «zen » du bonheur humain.

51. Wander, Brunnen, p. 92.

52. Wander, Baalbek, p. 184-185.

53. Connaissances tirées de Eugen Herrigel (1884-1955), Zen in der Kunst des Bogenschießens. Cet ouvrage avait paru la première fois à Constance en 1948 et fut réédité à partir de 1951 maintes fois à Munich au Barth Verlag et connut depuis d'innombrables rééditions - la dernière que nous ayons trouvée est la $46^{\mathrm{e}}$ édition de 2008. Wander avait utilisé la $16^{\mathrm{e}}$ édition de 1973 . L'ouvrage a été traduit en plusieurs langues dont en français en 1961 - cette édition enrichie de notes posthumes - et réédité en 1997 (La Voie du zen chez Le Grand Livre du mois). - Herrigel, professeur de philosophie formé à Heidelberg, avait passé quelques années au Japon (1924-1929) à l'Université de Sendai. Il s'y était formé au bouddhisme - ou ce qu'il pensait être le bouddhisme, car il ne lisait ni ne comprenait le japonais. Il avait tenté une synthèse entre cette approche et le mysticisme de Meister Eckhart, synthèse qui le poussa dans les bras des nazis. Plusieurs écrits entre 1934 et 1945 l'attestent, tout comme son adhésion au parti en 1937 et sa nomination comme recteur à Erlangen à la fin de la guerre. Wander, bien entendu, ne pouvait détenir ces informations lorsqu'il s'est construit sa propre interprétation du bouddhisme sur la base d'une déformation de celle-ci - voulue ou non. Pour informations complémentaires, consulter : [http:/www.univie.ac.at/rel_jap/an/Grundbegriffe/ Stereotype/Herrigels_Zen]. 
La France, à ce titre, est pour lui le pays qui présente le potentiel le plus important. À cela il trouve plusieurs raisons : la dictature de Vichy fut bel et bien incapable d'imposer la même discipline que les nazis - les Français y semblent réfractaires et surent opposer résistance active et passive ; l'administration se caractérise par une inefficacité flagrante ; l'appareil répressif est moins violent, de loin, que celui des Allemands : arrêté à maintes reprises, les gardes mobiles le laisseront filer - paresse ou négligence, peu importe ; le peuple aspire à un retrait dans une zone de confort personnel, incompatible avec un engagement entier à des fins de transformations de société - de quelque bord que celles-ci viennent. Certaines des apories françaises qui sont de fait, aux yeux de Wander, des points forts de cohésion sociale, sont la base humaine d'une société éloignée de toute dictature politique ou consumériste. Le Wander des années 1970 et 1980 voit dans une vie de marginal, de hippie, en Provence, le modèle de société susceptible de gagner son adhésion. Vivre en harmonie avec la nature, en liberté, à rythme posé et dans la frugalité, c'est ce dont il rêve, rêve qu'il ne réalisera pas.

\footnotetext{
Hippies hatten das Haus und das Grundstück gekauft, hatten einen Sommer dort verbracht, dann waren sie angeblich weitergezogen. Das Haus und den steilen, von stachligen Maquis gepflasterten Hang, auf dem das Haus versteckt liegt, überließen sie ihren Freunden. Sie züchten zu jeder Zeit dort oben wilde Menschenkinder, einen Haufen Katzen, Hühner, Ziegen und Flöhe. [...] Dort hielten sich jahrelang ein paar junge Kerle verborgen, baumlange Neger, amerikanische Deserteure, Männer mit Bärten, die ihre Militärpapiere verbrannt hatten und nun Zen studierten, die Bibel, Freud, Wilhelm Reich, Sartre, Heidegger, Engels und Marx ${ }^{54}$.
}

Nous pourrions multiplier les exemples tirés du Voyage provençal ou de La bonne vie. Le souhait de Wander de s'y installer ne verra jamais sa réalisation : jamais le Schlemihl n'arrivera à bon port.

\section{Métaphores et mythes dans l'œuvre de Fred Wander}

Nous avons vu que dans les ouvrages consultés, le mythe du Schlemihl et du Juif errant occupent une place centrale. Nous pouvons constater de même que ces deux mythes - devenus un seul par volonté fusionnelle de Wander - ne fonctionnent pas de manière complètement décontextualisée : le Schlehmil-paria est paria par rapport à certaines conditions de vie données : l'antisémitisme à Vienne, la condition d'émigré juif en France, la persécution nazie ou vichyste, le statut de communiste juif dans la Vienne d'après 1945, celui d'écrivain juif hétérodoxe viennois en RDA... Le Juif errant devient ainsi la métaphore d'une condition individuelle, certes, mais en même temps d'une condition de marginalité permanente de tout un groupe de population, celle des Juifs d'Europe dont l'assimilation a connu un échec brutal et souvent mortel par le fait des pouvoirs politiques totalitaires qui décidèrent de les marginaliser, de les expulser ou, pire encore, de les exterminer. Bien entendu, de telles conditions de vie cumulées rendent difficile la conception de projets biographiques qui puissent aller, souvent, au-delà de la sauvegarde d'un minimum d'intégrité physique : que Wander

54. Wander, Reise, p. 87/88. 
ait donné pour sous-titre à ses souvenirs autobiographiques « Von der Fröhlichkeit im Schrecken » est à ce titre révélateur d'une tentative quasiment désespérée de résilience face à une histoire dont on n'imagine pas l'horreur qu'elle génère.

De nombreux auteurs ont signalé la fonction du mythe comme tentative narrative de maîtriser l'horreur inexplicable qui vient s'abattre sur une partie de l'humanité. Wander a recours à certains « classiques » de la mythologie, aussi bien d'origine gréco-latine que judéo-biblique. S'il est inutile de revenir sur le mythe de Job qui dans la littérature antinazie occupe une place centrale, celle du Juif errant peut paraître plus surprenante en raison de son utilisation par la propagande antisémite, mais, comme le signale Blumenberg :

\begin{abstract}
Mythen sind Geschichten von hochgradiger Beständigkeit ihres narrativen Kerns und ebenso ausgeprägter marginaler Variationsfähigkeit. Diese beiden Eigenschaften machen Mythen traditionsgängig. [...] Mythen sind daher nicht so etwas wie " heilige Texte », an denen jedes Jota unberührbar ist. Geschichten werden erzählt, um etwas zu vertreiben. Im harmlosesten, aber nicht unwichtigsten Falle: die Zeit. Sonst und schwererwiegend: die Furcht ${ }^{55}$.
\end{abstract}

Sous cet angle, il nous semble plausible de considérer le Schlemihl comme métaphore renvoyant au Juif errant qui, lui-même, chez Wander, débarrassé de sa charge antisémite, est la métaphore d'une identité juive affirmée renvoyant au mythe de l'utopie eschatologique. Il devient, sous la plume de Wander, le messager messianique qui transcende oppression dictatoriale ou consumériste pour tenter de trouver ici-bas un présent meilleur fait d'harmonie de l'homme avec lui-même, entre les hommes et de ceux-ci avec la nature.

La France est dans ce contexte suffisamment éloignée de la RDA, géographiquement et politiquement, pour servir d'espace de projection à cette transcendance utopiste. Cependant, ne nous méprenons pas : la France qu'il prend en considération pour son projet est éloignée des hauts-lieux de la " civilisation » citadine. Il s'agit de coins reculés du Massif des Maures, en Ardèche, une île sur la côte - en cela son rêve est relativement banal car il correspond à ce que des milliers de «Aussteiger » firent pendant les années soixante et soixante-dix. D'ailleurs, au fur et à mesure de ses voyages, il put constater que les paysages qu'il avait adulés pendant sa période de vagabondage avant 1940 furent peu à peu saccagés par les développements industriels (étang de Berre) ou le tourisme de masse sur la côte languedocienne. La France telle qu'il la voit, est une France métaphorique servant de support narratif à son projet mythique plus individuel que social ou politique : comme Ulysse, ce Schlemihl-Juif-errant ne cesse de voyager, mais à la différence d'Ulysse il n'y a pas de retour pour lui car, s'il assume son identité, celle-ci n'a pas de nation-refuge à mettre à sa disposition : "Ich sage mir: Du wirst die Insel nicht finden, wenn Du nicht selbst die Insel bist im Lichte der Utopie ${ }^{56}$. » 
